\title{
Correction to: Role of androgen receptor splice variant-7 (AR-V7) in prostate cancer resistance to 2 nd-generation androgen receptor signaling inhibitors
}

Yezi Zhu • Susan L. Dalrymple • Ilsa Coleman · S. Lilly Zheng • Jianfeng Xu D • Jody E. Hooper • Emmanuel S. Antonarakis - Angelo M. De Marzo - Alan K. Meeker • Peter S. Nelson • William B. Isaacs • Samuel R. Denmeade · Jun Luo • W. Nathaniel Brennen (iD · John T. Isaacs (D)

Published online: 9 February 2021

(c) The Author(s), under exclusive licence to Springer Nature Limited 2021

Correction to: Oncogene

https://doi.org/10.1038/s41388-020-01479-6

Unfortunately, in the ESM of the original published version was the GEO accession number given incorrect.
The accession number for the RNA-seq data for the PDXs with the mouse genes subtracted is NCBI GSE149433 and for the LNCaP-95 and AR-KO cells is NCBI GSE 131985.

The original version was corrected. 